\title{
Acute hand ischemia following an accidental intra-arterial administration of dexamethasone and levetiracetam in a patient with superficial ulnar artery anomaly
}

\author{
Onur Saydam (1), Deniz Şerefli (D), Ayşen Yaprak Engin (D), Kadir Burhan Karadem (D), Ayşegül Kunt (1D \\ Department of Cardiovascular Surgery, Izmir Tepecik Training and Research Hospital, İzmir, Turkey
}

\begin{abstract}
Superficial brachioulnar artery (SUA) is an uncommon variation, having its course superficially to the flexors of the forearm muscles or may arise directly from the axillary or brachial arteries. Due to its superficial course, accidental intra-arterial cannulation may occur. Herein, we present a 55-year-old female case who had an accidental intra-arterial cannulation and received multiple intra-arterial injections of dexamethasone and levetiracetam. The symptoms of the patient mimicked an arterial embolism of the upper extremity and, therefore, the patient underwent an embolectomy procedure. In conclusion, upper extremity arterial anomalies and the possibility of accidental intraarterial cannulation should be kept in mind for acute ischemic cases.
\end{abstract}

Keywords: Iatrogenic disease; ischemia; superficial brachioulnar artery; ulnar artery.

Anatomically the ulnar artery is a terminal branch of brachial artery, originating from the cubital fossa. It passes medially deep to the median nerve and usually lies deep to the superficial flexor group of muscles of the forearm and lateral to the ulnar nerve. ${ }^{[1]} \mathrm{It}$, then, follows a subfascial course and forms the superficial palmar arch anterior to the flexor retinaculum. ${ }^{[2]}$ Superficial brachioulnar artery (SUA) is an uncommon variation with a prevalence of 0.7 to $7 \%$, and Dartnell et al. ${ }^{[3]}$ reported SUA in $4.2 \%$ of cases in their study. In this variation, SUA has its course superficial to the flexors of the forearm muscles and may arise directly from the axillary or brachial arteries. ${ }^{[4]}$ Due to its superficial course and close relationship with the basilic vein, it may complicate intravenous drug administration by accidental intra-arterial cannulation. ${ }^{[5]}$ Herein, we report an unusual case of accidental SUA cannulation and multiple intra-arterial injections of dexamethasone and levetiracetam, mimicking an arterial embolism of the upper extremity.

\section{CASE REPORT}

A 55-year-old female patient was under follow-up in the neurosurgery department due to an intracranial mass. She was, then, referred to the cardiovascular surgery department with severe acute left hand ischemia marked in the fourth and fifth digits, pain, and weakness. Color Doppler ultrasound (US) was performed and no flow was detected in the ulnar artery. Due to acute arterial symptoms and findings on Color Doppler US, the patient was admitted to the operation room with the diagnosis of ulnar artery embolism. A written informed consent was obtained from the patient.

Under local anesthesia, a $4 \mathrm{~cm}$ incision was made through the ventromedial forearm. During the routine brachial artery dissection in the cubital fossa, a rudimentary, non-pulsatile, superficial vessel was observed superficially to the flexors of the forearm muscles. There was a very small hematoma on the surface of the vessel which was thought to be the

Received: August 05, 2018 Accepted: September 03, 2018 Published online: September 07, 2018

Correspondence: Onur Saydam, MD. İzmir Tepecik Ë̆itim ve Araștırma Hastanesi Kalp ve Damar Cerrahisi Kliniği, 35180 Yenişehir, İzmir, Turkey. e-mail: onursaydam@hotmail.com 
cannulation entry. In the cubital fossa, the vessel crossed from medial to the lateral side. The brachial artery was underneath this vessel and terminated by dividing into the radial artery and common interosseous artery in the cubital fossa (Figure 1). The patient was diagnosed with SUA anomaly and under anticoagulation with 5,000 IU unfractionated heparin, arteriotomy was performed. Thrombectomy was also performed to the distal and proximal part of the SUA with $3 \mathrm{~F}$ and $4 \mathrm{~F}$ Fogarty catheters (Edwards Lifesciences, Irvine, CA, USA). There was no embolism material obtained; arterial pulse was restored and symptoms relieved. Subcutaneous enoxaparin $0.01 \mathrm{~mL} / \mathrm{kg}$ twice daily was initiated. Based on a more detailed medical history, it was found that cannulation was performed via the left arm. A total of $8 \mathrm{mg}$ of dexamethasone and $500 \mathrm{mg}$ of levetiracetam were administered as a bolus injection via this cannula twice a day for one week and the cannula was removed one day before the urgent vascular surgery due to malfunction.

The patient was referred to the neurosurgery department uneventfully on the postoperative second day. At one month, control contrast enhanced computed tomography (CT) showed SUA arising from the axillary artery and running superficially to the forearm flexor muscle, crossing from medial to lateral side in the cubital fossa. At the proximal one-third of the forearm, it was occluded. Distal two-third of the forearm ulnar artery was in normal position and the blood was supplied via the superficial palmar arch.

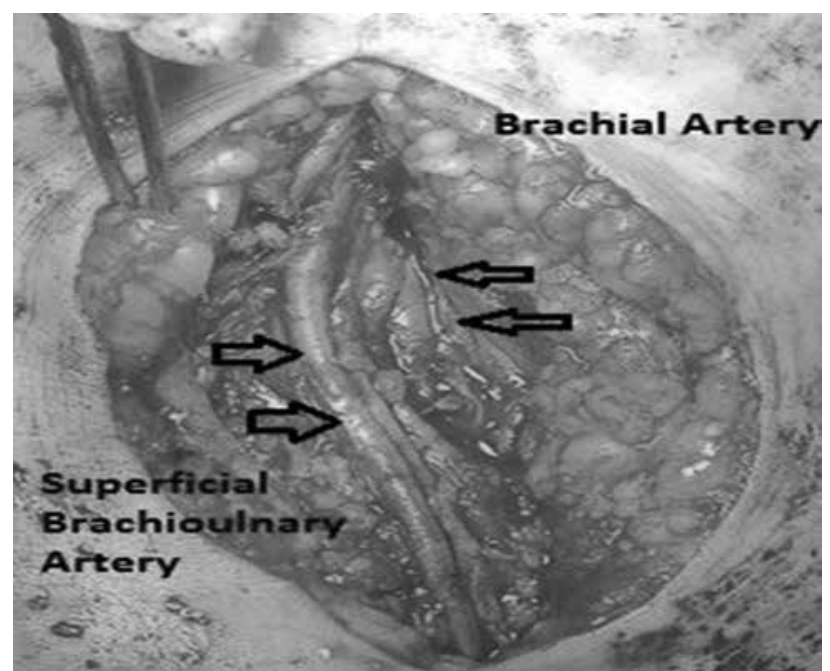

Figure 1. An intraoperative image of superficial brachioulnar artery and brachial artery located underneath this vessel in the cubital fossa.

\section{DISCUSSION}

Upper extremity anatomical variation has a high incidence up to $20 \% .{ }^{[6]}$ These anatomical variations are mostly named based on the regions of their location, such as brachial, ulnar, radial or a combination of these arteries and according to the superficial course of the vessel. ${ }^{[7]}$ The SUA is not common and vascular surgeons should recognize these variations to decrease morbidity and to perform a safe surgery. Similar to our case, the SUA can originate from the axillary artery (26.9\%) and join the ulnar artery at the level of the wrist. However, the SUA mostly originates from the brachial artery (73.1\%) and can totally replace the ulnar artery. ${ }^{[8]}$ Due to its superficial position, it is unguarded against traumas and accidental intraarterial cannulation, even for experienced physicians. ${ }^{[9]}$ Accidental intra-arterial injection of drugs can lead to intimal injuries, inflammation, and hemolysis by the drug crystalloids. Also, local intimal secretion disorders, such as norepinephrine, can cause arterial vasospasm leading to ischemia. ${ }^{[10]}$ The only sign of these disorders may be arterial ischemia with the symptoms of paresthesia, pain, pallor, pulseless, and paralysis. The dose of the drug is as important as the drug type. In our case, the patient received only two intravenous drugs, namely dexamethasone and levetiracetam. To the best of our knowledge, there are no controlled-randomized studies investigating intraarterial injection of dexamethasone or levetiracetam. In a review, Devulapalli ${ }^{[11]}$ reported that the most commonly injected substance was opiates; however, the patients who received benzodiazepine had the highest frequency of amputations (50\%). There is no standard treatment protocol for intra-arterial drug administration. ${ }^{[12]}$ The main goal of the treatment should be relieving the symptoms, precluding the arterial vasospasm, and restoring the arterial blood flow. ${ }^{[10]}$ Although we did not observe any thrombus material in our case, all the aforementioned alterations can lead to thrombosis. According to Sen et al., ${ }^{[12]}$ anticoagulation treatment is recommended as the initial treatment in all cases. Medical treatment may include nicardipine, reserpine, papaverine, pentoxifylline, steroids, ilioprost, and anticoagulation and thrombolytic therapies. Due to the lack of evidence, there is no specific medical treatment option. ${ }^{[1]}$ Surgical sympathectomy and thrombectomy can be considered surgical treatments. ${ }^{[10]}$ Endovascular treatment, such as catheter-directed thrombolysis, may be also a treatment of choice. ${ }^{[13]}$ In our case, however, the patient was followed in the neurosurgery department due to an intracranial mass and this 
can be considered an absolute contraindication for thrombolysis treatment. To prevent inadvertent intraarterial cannulation, we should keep in mind that arterial cannulation is always probable in the regions of arterial trace. We also should check the manual palpation of the vessel and ensure that there is no pulsatile movement of blood through the cannula. Distal ischemia and more severe pain can be the signs of inadvertent intra-arterial cannulation. ${ }^{[14]}$

In conclusion, the possibility of accidental intraarterial cannulation should be kept in mind in patients with upper extremity arterial anomalies. As in the present case, anatomic variations can be overlooked using CDU and, therefore, preoperative $\mathrm{CT}$ or angiography should be performed in eligible cases.

\section{Declaration of conflicting interests}

The authors declared no conflicts of interest with respect to the authorship and/or publication of this article.

\section{Funding}

The authors received no financial support for the research and/or authorship of this article.

\section{REFERENCES}

1. Solan S. Accessory superficial ulnar artery: a case report. J Clin Diagn Res 2013;7:2943-4.

2. Vollala VR, Jetti R, Soni S. High origin of an ulnar artery-development and surgical significance. Chang Gung Med J 2011;34(6 Suppl):39-42.

3. Dartnell J, Sekaran P, Ellis $H$. The superficial ulnar artery: incidence and calibre in 95 cadaveric specimens. Clin Anat 2007;20:929-32.

4. Natsis K, Papadopoulou AL, Paraskevas G, Totlis T, Tsikaras P. High origin of a superficial ulnar artery arising from the axillary artery: anatomy, embryology, clinical significance and a review of the literature. Folia Morphol (Warsz) 2006;65:400-5.

5. McWilliams RG, Sodha I. Doppler ultrasound diagnosis of a superficial ulnar artery. Eur J Ultrasound 2000;12:155-7.

6. Rodríguez-Niedenführ M, Burton GJ, Deu J, Sañudo JR. Development of the arterial pattern in the upper limb of staged human embryos: normal development and anatomic variations. J Anat 2001;199(Pt 4):407-17.

7. Rodríguez-Niedenführ M, Vázquez $\mathrm{T}$, Nearn L, Ferreira B, Parkin I, Sañudo JR. Variations of the arterial pattern in the upper limb revisited: a morphological and statistical study, with a review of the literature. J Anat 2001;199(Pt 5):547-66.

8. Senanayake KJ, Salgado S, Rathnayake MJ, Fernando $\mathrm{R}$, Somarathne K. A rare variant of the superficial ulnar artery, and its clinical implications: a case report. J Med Case Rep 2007;1:128.

9. Chin KJ, Singh K. The superficial ulnar artery--a potential hazard in patients with difficult venous access. Br J Anaesth 2005;94:692-3.

10. Lehavi A, Sandler A, Netzer A, Katz YS. Vascular injury following accidental intra-arterial injection of clindamycin: adverse drug reaction report. Minerva Anestesiol 2011;77:468-9.

11. Devulapalli C, Han KD, Bello RJ, LaPorte DM, Hepper $\mathrm{CT}$, Katz RD. Inadvertent Intra-Arterial Drug Injections in the Upper Extremity: Systematic Review. J Hand Surg Am 2015;40:2262-2268.e5.

12. Sen S, Chini EN, Brown MJ. Complications after unintentional intra-arterial injection of drugs: risks, outcomes, and management strategies. Mayo Clin Proc 2005;80:783-95.

13. Breguet R, Terraz S, Righini M, Didier D. Acute hand ischemia after unintentional intraarterial injection of drugs: is catheter-directed thrombolysis useful? J Vasc Interv Radiol 2014;25:963-8.

14. Ghouri AF, Mading W, Prabaker K. Accidental intraarterial drug injections via intravascular catheters placed on the dorsum of the hand. Anesth Analg 2002;95:487-91. 